\title{
Change of optical properties of hair cells during malignant tumour development
}

\author{
V.S. Maryakhina ${ }^{1 *}$, L.S. Scheglova ${ }^{1}$, K.A. Anenkova ${ }^{2}$ \\ ${ }^{1}$ Orenburg state university, 13 Pobedy st., Orenburg, 460018, Russia \\ ${ }^{2}$ M. V. Lomonosov Moscow state university, 1 Leninskiye Gory, Moscow, 119991, Russia \\ * e-mail: v.s.maryakhina@gmail.com
}

\begin{abstract}
The results of experimental investigations on the differences of wool microstructure and its biophysical properties of healthy mice and mice with malignant tumour are represented in the paper. It is shown that destruction of wool structure as well as biochemical composition change of wool cells is during pathology development. Difference is seen even when tumour is small. We have shown that the general regularity of spectral measurements for mammary and hair cells is conserved at health state and pathology present. Taking into account this, the represented data about wool structure can be used to improve the optical techniques for non-invasive diagnostics of cancer diseases at different stages of pathology. (C) 2015 Samara State Aerospace University (SSAU).
\end{abstract}

Keywords: breast cancer; hair; optical biomedical diagnostics; dynamic light scattering; fluorescence.

Paper \#1868 received 2014.11.29; revised manuscript received 2015.01.13; accepted for publication 2015.01.16; published online 2015.03.28.

\section{References}

1. V. V. Tuchin, "Light scattering study of tissues," Phys. Usp. 40, 495 - 515 (1997).

2. V. S. Maryakhina, and V. V. Gun'kov, "Fluorescent probe immobilization into enzyme molecules", Computer research and modeling 5, 201-208 (2013).

3. S. N. Letuta, V. S. Maryakhina, and R. R. Rakhmatullin, "Optical diagnostics of biological tissue cells during their cultivation in polymers," Quantum electronics 41, 314-317 (2011).

4. Th. Schlake, "Determination of wool structure and shape," Seminars in Cell \& Developmental Biology 18, 267-273 (2007).

5. S. B. Park, S. W. Choi, and A. Y. Nam, "Wool Tissue Mineral Analysis and Metabolic Syndrome," Biol Trace Elem Res 130, 218-228 (2009).

6. V. S. Maryakhina, L. S. Scheglova, and K. A. Anenkova, "Features of structure of external layer of murine hair at different stages of malignant tumour development," Journal of Innovative Optical Health Sciences 8, 1541001 (2015).

7. G. Z. Kammins, and E. R. Paik, Photon Correlation and Light Beating Spectroscopy. New York: Plenum (1974).

8. R. Pecora, Dynamic Light Scattering from Macromolecules, Stanford: Stanford Univ. (1984).

9. K. A. Anenkova, G. P. Petrova, V. V. Gibizova, L. A. Osminkina, and K. P. Tamarov, "Optical properties of serum albumin water solutions, containing mesoporous silicon particles," Optics and Spectroscopy 115, 166$170,(2013)$.

10. G. E. Dobretsov, Fluorescent Probes in the Studies of Cells, Membranes, and Lipoproteins, Moscow: Nauka (1989).

11. L. S. Scheglova, L. L. Abramova, and V. S. Maryakhina, "Optical diagnostics of tumour cells at different stages of pathology development," Quantum electronics 43, 1088-1090 (2013).

12. N. M. Emanuel, R. E. Kavetskiy, B. N. Tarusov, and E. P. Sidorik, Biophysics of cancer, Kiev: Science (1976). 
13. S. N. Letuta, and V. S. Maryakhina, "The delayed fluorescence kinetics as a method of biological tissue diagnostics," Proc. SPIE 7999, 799907 (2011).

14. V. James, J. Kearsley, T. Irving, Y. Amemiya, and D. Cookson, "Using wool to screen for breast cancer," Nature 398, 33-34 (1999).

15. F. Briki, B. Busson, B. Salicru, F. Estève, and J. Doucet, "Breast-cancer diagnosis using wool," Nature 400, 226 (1999).

16. M. Hart, "Using wool to screen for breast cancer," Synchrotron Rad. News 12, 31-37 (1999).

17. Q. Pasha, S. A. Malik, N. Shaheen, and M. H. Shah, "Investigation of trace metals in the blood plasma and scalp wool of gastrointestinal cancer patients in comparison with controls," Clinica Chimica Acta 411, 531$539(2010)$.

18. E. L. Abel, J. M. Angel, K. Kiguchi, and J. DiGiovanni, "Multi-stage chemical carcinogenesis in mouse skin: Fundamentals and applications," Nature Protocols 4, 1350-1362 (2009).

19. V. S. Maryakhina, and S. N. Letuta, "Pathology development stage and its influence to the delayed fluorescence kinetics of molecular probes," Laser physics 23, 025604 (2013).

\section{Introduction}

The optical techniques of biomedical diagnostics are used for detection of biotissue pathological states due to their non-invasiveness and high sensitivity to pathology. However, sensitivity of all these techniques is not enough for early diagnostics of cancer diseases. According to that it is essential to raise the accuracy of the optical methods. Parameter, which significant different for health and tumour cells, should be found. It can be carried out by either device improving or research object change.

Biotissues and somatic cells are widespread objects for the optical biomedical diagnostics but their significant heterogeneity together with high scattering light [1] significantly complicate obtaining statistically reliable data. That's why new research objects are needed.

In this work we show the possibility of using hair for optical biomedical diagnostics because of difference in their structure in normal and pathological states.

\section{Objects and methods of research}

\subsection{Objects of research}

The object of the research was wool of mice (line BYRB) with spontaneous tumours of mammary gland. Wool had been cut from all skin surfaces of healthy mice and mice with malignant tumour(s) of same line. Additionally, we had also carried out experiments with wool from skin located above tumour only. Diameter of tumour capsule was from 1 to $3 \mathrm{~cm}$. The wool aliquot was previously cleared twice in medical alcohol. To obtain reliable results not less than 15 female mice were used for each type of samples.

\subsection{Optical microscopy}

The cleared wool was placed on the slide. Wool structure was investigated by the optical inverted microscope Altami invert 3 with video camera working in phase contrast mode (magnification $\mathrm{x} 400$ ).

\subsection{Spectrofluorimetry}

The cleared wool aliquots were placed into collagenase solution $(0.5 \mathrm{mg} / \mathrm{ml})$ dissolved in phosphate buffer $(\mathrm{pH}=7.4)$ and were incubated at $37{ }^{\circ} \mathrm{C}$ for 40 minutes for stirring. The obtained suspension (collagenase with dissolved cells and peptides) was investigated by spectrofluorimetry. A few microliters of rhodamine $6 \mathrm{G}$ was added to the suspension and was incubated during 10-15 minutes for diffusion equilibrium establishment [2]. The colored suspension was placed to slide for full drying up. Fluorescence spectra were measured on the optical device (fig.1) containing xenon lamp XBO $150 \mathrm{~W} / 1$ (light source) $\mathbf{1}$ and monochromator MDR-206 2. The monochromatic light was focused by the lens $\mathbf{3}$ and was routed to sample surface 5 by the swivel mirror 4. Emission of the samples was focused by the lens $\mathbf{6}$ to the entrance slit of the monochromator MDR-41 7 and was registered by FEU-100 8. Collection, accumulation and processing of the experimental data were performed in an automatic mode by the personal computer and CAMAC crate 9 and computer 10. The excitation wavelength was $515 \mathrm{~nm}$.

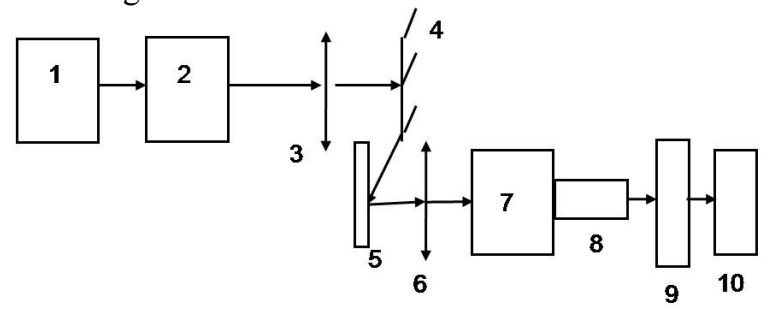

Fig. 1 Scheme of experimental device for fluorescence spectra measurements.

\subsection{Phosphorescence kinetics}

The obtained cell suspension was cleared twice from collagenase molecules by centrifuging at $6000 \mathrm{rpm}$ for 5 minutes. Aliquot of bengal rose was added to the cell solution and was incubated during 10-15 minutes. The colored cells were placed to the mica for measuring. Phosphorescence kinetics was measured on experimental setup for biomedical investigations. Its 
description had been published in detail in our earlier work [3]. The wavelength of excitation was at $532 \mathrm{~nm}$ (the second harmonics of Nd:YAG laser). Registration of emission was at $680 \mathrm{~nm}$. All measurements were carried out at atmosphere pressure. The registered signal is result of accumulation of 20 signals from each sample for measurements error decrease.

\subsection{Dynamic light scattering}

The obtained cell suspension was placed to "Photocor complex" for cells size measuring. Laser wavelength was $647 \mathrm{~nm}$, it power was $25 \mathrm{~mW}$. Each measurement was during $10 \mathrm{~min}$. For statistical adequacy the all represented results are average of ten or more measurements. All used solutions were free-prepared from dry compounds produced by Sigma-aldrich.

\section{The results and discussion}

It is known [4] that hair of both animals and people has layer structure. External layer is connective tissue cells. Next two internal Henley and Huxley layers consist of keratinized cells with irregular shape. The internal layer is hair shaft. In spite of complex multilayer structure, hair can be as health indicator sometimes [5]. Any changes of metabolic processes lead to change of wool biochemical composition. As a rule, its destruction starts from external layer. Photo samples of the wool of health mice females and females with spontaneous tumour of mammary gland are presented in Figure 2. As you can see, disturbance of hair structure is traceable on sequential stages. Wool of health mouse has dense structure, external layer is pronounced. At the time, wool of mice with tumour is thinner, external and internal layers are destroyed. Difference is even when tumour diameter is small.

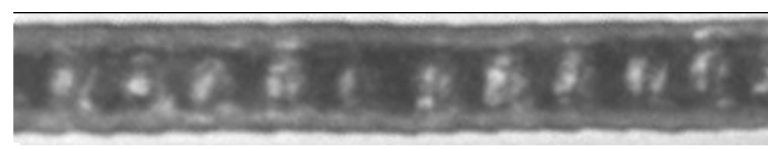

a)

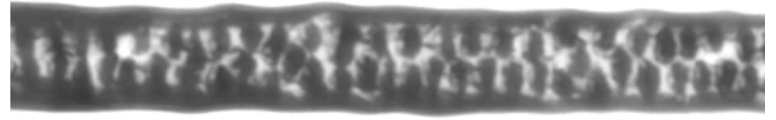

b)

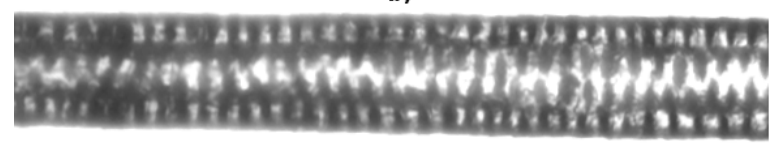

c)

Fig. 2 Wool structure of health mouse (a) and mice with tumour $1 \mathrm{~cm}$ (b), $3 \mathrm{~cm}$ (c). Magnification x400.

The described changes lead to absorption spectra changes described in our previous work [6]. A serial decrease of supernatant optical density is. At the time, their maxima spectra shift to short-wave region. It is a result of hair structure destruction with pathology development, cells number decreases.
In addition to the decrease of cell quantity in the external layer, its thinning was observed. These data were confirmed by dynamic light scattering method (DLS). The DLS method allows to determine the translation diffusion coefficient of disperse particles in a fluid through the analysis of the characteristic time of fluctuations of the scattered light intensity [7]. The chaotic Brownian motion of dispersed particles causes microscopic fluctuations of their local concentration. These fluctuations, in turn, lead to local inhomogeneities of the refractive index. The passage of a laser beam through the medium means that part of the light is scattered on these inhomogeneities; the fluctuations of the intensity of the scattered light correspond to fluctuations of the dispersed particles' local concentration.

The dynamic information in the particles is derived from autocorrelation of the intensity trace recorded during the experiment. The autocorrelation function describes the relationship between the signal at the delay time $\tau$ and $\tau+\mathrm{d} \tau$ as follows:

$$
G(t)=\langle I(t) \cdot I(t+\tau)\rangle,
$$

where $\tau$ is the delay time and I is the density.

This technique is named photon correlation spectroscopy and it is also known as quasielastic light scattering [8].

In the case of a mono-dispersed solution, that contains spherical non interacting particles of the same size, it is possible to show that the photocurrent power spectrum has the form of a Lorentzian curve [7] with half width $\Gamma$. The normalized intensity correlation function is described by an exponential with relaxation time $\tau_{\text {rel }}=1 / \Gamma$ as

$$
\mathrm{g}_{1}(\tau)=\exp (-\Gamma \mathrm{t})
$$

The decay rate $\Gamma$ is related to the physical properties of the particles and the experimental conditions by the following expressions:

$$
\begin{aligned}
& \Gamma=\mathrm{D}_{\mathrm{t}} \mathrm{q}^{2}, \\
& \mathrm{D}_{\mathrm{t}}=\frac{\mathrm{kT}}{6 \pi \eta \mathrm{R}}, \\
& \mathrm{q}=\mathrm{k}_{\mathrm{i}}-\mathrm{k}_{\mathrm{s}}=\left(\frac{4 \pi \mathrm{n}}{\lambda}\right) \sin \frac{\theta}{2},
\end{aligned}
$$

where $k_{i}$ is the wave vector of incident radiation, $k_{s}$ is the wave vector of scattered radiation, $D_{t}$ is the translational diffusion coefficient of particles, $\mathrm{n}$ is the refractive index of a medium, $\lambda$ is the scattered light wave length, $\theta$ is the scattering angle, $T$ is the absolute temperature of the scattering solution, $K$ is the 
Boltzman's constant, $\eta$ is the solvent viscosity, and $R$ is the hydrodynamic radius of a particle.

In the case of polydisperse solutions with different sizes of particles, the photocurrent spectrum is a continuous set (integral) of Lorentzian curves with different half widths. Therefore, to find the size distribution of particles (diffusion coefficients); it is necessary to solve the inverse spectral problem in the form of anintegral equation with the Lorentzian kernel:

$$
\begin{aligned}
& g^{(2)}(t)=\left(g^{(1)}(t)\right)^{2}+1+\zeta(t) \\
& g^{(1)}(t)=\int_{0}^{\infty} P(\Gamma) \exp (-\Gamma t) d \Gamma
\end{aligned}
$$

where $g^{(1)}(t)$ is the normalized autocorrelation function of a signal, $g^{(2)}(t)$ is the normalized autocorrelation function of intensity, and $\zeta(t)$ is an error associated with the stochastic nature of the signal itself rather than with the inaccuracy of photocurrent measurement or the noises of a recording channel $[7,9]$.

Equation (7) is known as the Siegert relation, will allow one to calculate $g^{(1)}(t)$ in terms of $g^{(2)}(t)$ accumulated by a correlator in the course of an experiment. Integral equation (3) forms the main principle of data processing in the method of photon correlation spectroscopy.

The results of our experiments were processed using the DYNALS package of programs, in which approximate solution (3).

The results of calculations are presented in table 1 . As you can see each sample contains two cell types. These cell types have different size. We assume that it connects on partially separation of Henley layer during collagenase treatment. With pathology process development cells size decrease for both cell types. We note that difference between wool cell size of normal states and small tumour are nearly $36 \%$ for two cell types. Consequently, these distinctions are statistically significant. We do not observed difference in the results after investigation of wool cuted from all skin surface and from tumour surface only for all mice types.

Moreover, biochemical composition is also changed during breast cancer development. It is confirmed by fluorimetric measurements with molecular probes. It is known [10] that rhodamine $6 \mathrm{G}$ penetrates into cell and connects with mitochondria. Thus, fluorescence spectra of rhodamine can be as an indicator of mitochondria structure changes. In Fig. 3 fluorescence spectra of the probe are depicted. They shift to long wave region with tumour development. This regularity for rhodamine we had also seen for normal and cancer mammary cells. These results had been published in our previous work [11]. Wave lengths are different in two types of experiments because of difference of cells biochemical composition, however, the general regularity is observed.

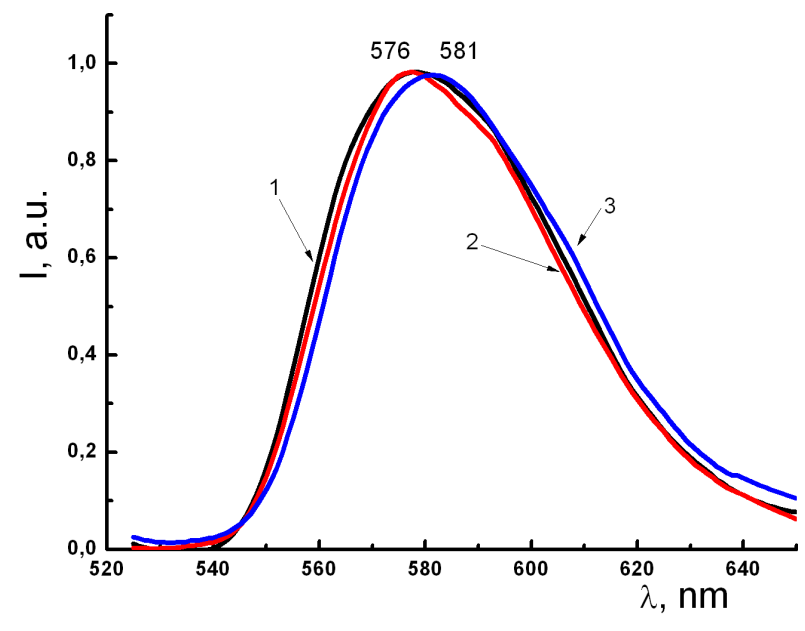

Fig. 3 Fluorescence spectra of rhodamine $6 \mathrm{G}$ in hair cells from health mouse (1), from mouse with small (2) and large tumour (3).
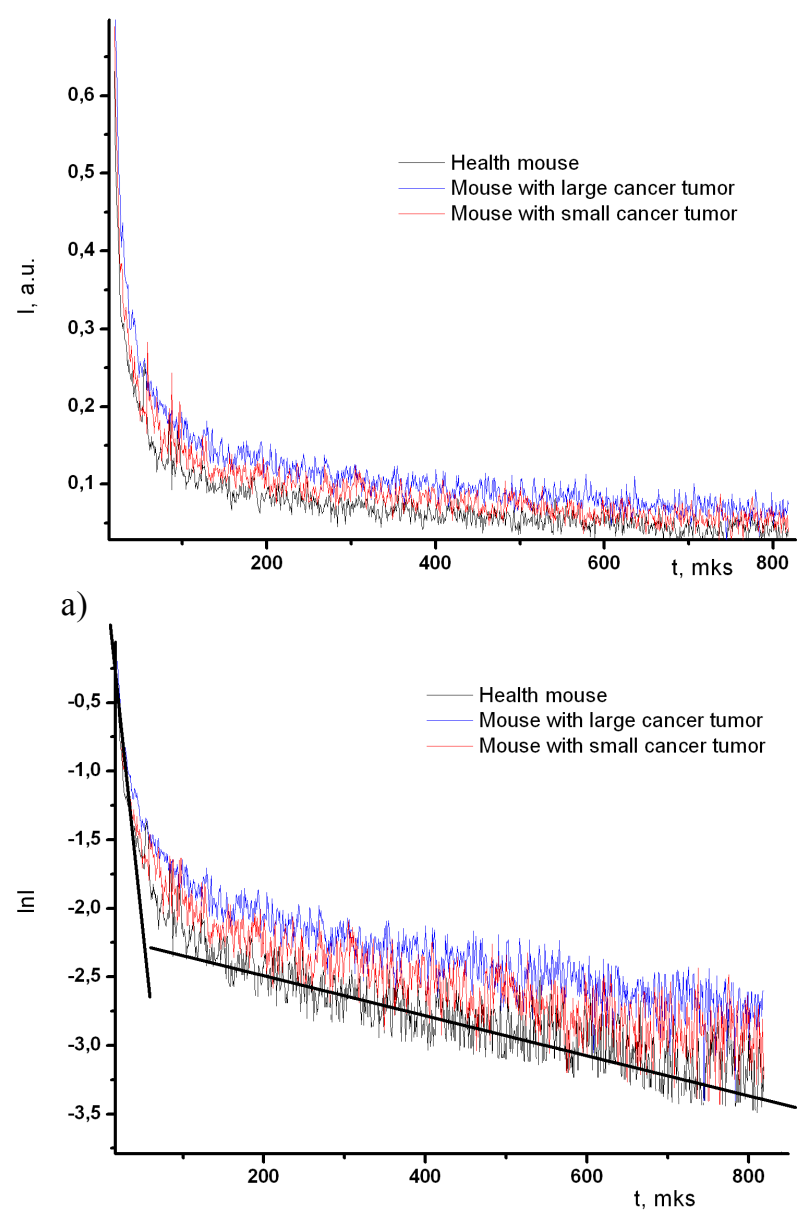

b)

Fig. 4 The phosphorescence kinetics of bengal rose located in wool cells from health mouse (1), from mouse with small (2) and large tumour (3). Curves are carried out in Descartes (a) and semilogarithmic (b) coordinates.

The microviscosity and peptide concentrations of hair cells are also changes with tumour growth. It can be measured by phosphorescence kinetics of bengal rose 
(Fig. 4a) which connect with peptides. The kinetics has a two-exponential type. It is distinctly seen if these curves are carried out in semilogarithmic coordinates (Fig. 4b).

These curves of Fig. 4a are approximated by the following function

$$
I_{p h}(t)=A_{1} \exp \left(-t / \tau_{1}\right)+A_{2} \exp \left(-t / \tau_{2}\right)
$$

Value $\tau_{1}$ and $\tau_{2}$ are represented in table 2. As you can see from approximation results, $\tau_{1}$ decreases with tumour growth. It can be results of increases of annihilation processes rate with triplet states of the probe. At the time $\tau_{2}$ rises with pathology development. It can be connected with peptide concentration increases [12] and immobilization of the probe into peptide molecules. We note that similar regularity had been seen for fluorescent probes located in normal and cancer mammary cells [13].

\section{Conclusion}

Although biomedical diagnostics methods are present, search of method of the earlier diagnostics of cancer diseases is. For example, earlier [14] the method of breast cancer diagnostics based on wool structure investigation by X-rays was proposed. Later investigations $[15,16]$ had not confirmed these data. Nonetheless, research of wool structure for diagnostics applications are carrying on (see, for example, [17]).

The biological tissue cells are objects with specific very inhomogeneous structure. Effectivity of photoprocesses happening inside the cells depends on composition of intracellular structures, composition of cell membrane etc. In its turn, the physical-chemical composition of the cells received from pathogenic tissues depends on the stage of pathological process development [18]. It leads to change spectralluminescent properties of cells obtained from tumour at different stages of pathology as well as photosensitizers located within it $[11,19]$. In the paper we have shown that the general regularity of spectral measurements for mammary and hair cells is conserved at health state and pathology present. Taking into account this, the represented data about wool structure can be used to improve the optical techniques for non-invasive diagnostics of cancer diseases at different stages of pathology.

The work was partially supported by grant of Ministry of science and education of Russian Federation № 450 from 01.02.2014

Table 1 Cells radius of external wool layer and their diffusion coefficients.

\begin{tabular}{ccccccc}
\hline \multicolumn{2}{c}{ Normal wool } & \multicolumn{2}{c|}{$\mathbf{d}_{\text {tumour }}=\mathbf{1} \mathbf{~ c m}$} & \multicolumn{2}{c}{$\mathbf{d}_{\text {tumour }}=\mathbf{3} \mathbf{~ c m}$} \\
\hline $\begin{array}{c}\mathbf{R}, \\
\mathbf{n m}\end{array}$ & $\begin{array}{c}\mathbf{D}_{\mathbf{t}} \cdot \mathbf{1 0}^{-\mathbf{8}}, \\
\mathbf{c m}^{2} / \mathbf{s}\end{array}$ & $\begin{array}{c}\mathbf{R}, \\
\mathbf{n m}\end{array}$ & $\begin{array}{c}\mathbf{D}_{\mathbf{t}} \cdot \mathbf{1 0}^{-8}, \\
\mathbf{c m}^{2} / \mathbf{s}\end{array}$ & $\begin{array}{c}\mathbf{R}, \\
\mathbf{n m}\end{array}$ & $\begin{array}{c}\mathbf{D}_{\mathbf{t}} \cdot \mathbf{1 0}^{-\mathbf{8}}, \\
\mathbf{c m}^{2} / \mathbf{s}\end{array}$ \\
\hline $776 \pm 146$ & $0,28 \pm 0,06$ & $495 \pm 83$ & $0,44 \pm 0,07$ & $368 \pm 74$ & $0,59 \pm 0,13$ \\
$199 \pm 36$ & $1,1 \pm 0,2$ & $126 \pm 13$ & $1,7 \pm 0,2$ & $99.4 \pm 13$ & $2,7 \pm 0,4$ \\
\hline
\end{tabular}

Table 2 The result of approximation of bengal rose phosphorescence curves by function (8).

\begin{tabular}{lcc}
\hline \multicolumn{1}{c}{ Curves } & $\boldsymbol{\tau}_{\mathbf{1}}, \boldsymbol{\mu s}$ & $\boldsymbol{\tau}_{\mathbf{2}}, \boldsymbol{\mu s}$ \\
\hline $\begin{array}{l}\text { Health mouse } \\
\text { Mouse with }\end{array}$ & $19.3 \pm 0.5$ & $657 \pm 11.5$ \\
small tumour & $16.8 \pm 0.4$ & $673 \pm 13.1$ \\
$\begin{array}{l}\text { Mouse with large } \\
\text { tumour }\end{array}$ & $16.1 \pm 0.3$ & $727 \pm 14.1$ \\
\hline
\end{tabular}

